\title{
The effects of visuomotor calibration to the perceived space and body, through embodiment in immersive virtual reality.
}

\author{
ELENA KOKKINARA ${ }^{\mathrm{a}, \mathrm{b}, \mathrm{c}}$, MEL SLATER ${ }^{\mathrm{a}, \mathrm{c}, \mathrm{d}}$ and JOAN LÓPEZ-MOLINER ${ }^{\mathrm{b}, \mathrm{c}}$, \\ ${ }^{a}$ EVENT Lab, Facultat de Psicologia, Universitat de Barcelona, Barcelona, Spain \\ ${ }^{b}$ VISCA lab, Departament de Psicologia Básica, Universitat de Barcelona, Spain \\ ${ }^{\mathrm{c}}$ Institute for Brain, Cognition and Behaviour (IR3C), Spain \\ ${ }^{\mathrm{d}}$ Instituci Catalana Recerca i Estudis Avancats (ICREA), Barcelona, Spain
}

We easily adapt to changes in the environment that involve cross-sensory discrepancies (e.g. between vision and proprioception). Adaptation can lead to changes in motor commands so that the experienced sensory consequences are appropriate for the new environment (e.g. we program a movement differently while wearing prisms that shift our visual space). In addition to these motor changes, perceptual judgments of space can also be altered (e.g. how far I can reach with my arm?). However, in previous studies that assessed perceptual judgments of space after visuomotor adaptation, the manipulation was always a planar spatial shift, whereas changes in body perception could not directly be assessed. Here, we investigated the effects velocitydependent (spatiotemporal) and spatial scaling distortions of arm movements on space and body perception, taking advantage of immersive virtual reality. Exploiting the perceptual illusion of embodiment in an entire virtual body, we endowed subjects with new spatiotemporal or spatial 3D mappings between motor commands and their sensory consequences. The results imply that spatiotemporal manipulation of 2 and 4 times faster can significantly change participants' proprioceptive judgments of a virtual object's size, without affecting the perceived body-ownership, though affecting agency of the movements. Equivalent spatial manipulations of 11 and 22 degrees of angular offset also had a significant effect on the perceived virtual object's size, however the mismatched information did not affect either the sense of body-ownership or agency. We conclude that adaptation to spatial and spatiotemporal distortion can similarly change our perception of space, although spatiotemporal distortions can more easily be detected.

Categories and Subject Descriptors: H.5.1 [Information Interfaces and Presentation]: Multimedia Information SystemsArtificial, Augmented and Virtual Realities, Evaluation/methodology; I.3.7 [Computer Graphics]: Three-Dimensional Graphics and Realism-Virtual reality

General Terms: Experimentation, Human Factors

Additional Key Words and Phrases: body-ownership, sensorimotor adaptation, space perception, spatiotemporal distortions, virtual reality

\section{ACM Reference Format:}

Kokkinara, E., Slater, M. and López-Moliner, J. 2014. The effects of visuomotor calibration to the perceived space and body, through embodiment in immersive virtual reality. ACM Trans. Appl. Percept. 2, 3, Article 1 (May 2014), 23 pages.

DOI $=10.1145 / 0000000.0000000$ http://doi.acm.org/10.1145/0000000.0000000

This work was funded by the FP7 EU VR-HYPERSPACE (AAT-285681) project funded under the Aeronautics and Air Transport (AAT) workprogramme.

Author's address: Joan López-Moliner, Vision and Control of Action group and Departament de Psicologia Básica, Universitat de Barcelona, Campus de Mundet - Edifici de Ponent, Passeig de la Vall d'Hebron 171, 08035 Barcelona, Spain.email: j.lopezmoliner@ub.edu;

Permission to make digital or hard copies of part or all of this work for personal or classroom use is granted without fee provided that copies are not made or distributed for profit or commercial advantage and that copies show this notice on the first page or initial screen of a display along with the full citation. Copyrights for components of this work owned by others than ACM must be honored. Abstracting with credit is permitted. To copy otherwise, to republish, to post on servers, to redistribute to lists, or to use any component of this work in other works requires prior specific permission and/or a fee. Permissions may be requested from Publications Dept., ACM, Inc., 2 Penn Plaza, Suite 701, New York, NY 10121-0701 USA, fax +1 (212) 869-0481, or permissions@acm.org.

(c) 2014 ACM 1544-3558/2014/05-ART1 $\$ 15.00$

DOI 10.1145/0000000.0000000 http://doi.acm.org/10.1145/0000000.0000000 


\section{INTRODUCTION}

In order to program and execute goal-directed movements, we need to constantly update the changing position of our body or body-parts (e.g. limbs), using the sensory feedback. But what happens if vision and proprioception do not coincide, e.g. if we see our hand at a distorted position or moving with a different velocity to our intention? The brain will typically alter the motor commands (often unconsciously) in such a way that the shifted visual representation of the moving body part will reach the target location. This is known as visuomotor adaptation. Recent evidence supports the notion that this adaptive behaviour to spatial distortions can further affect our judgments of space perception and reaching behaviour, but whether this is also true for velocity-dependent (spatiotemporal) distortions has not previously been studied. Moreover, no clear evidence exists about whether spatial or spatiotemporal distortions can even affect our body perception. It is very difficult to observe such effects in reality, because of course our arm normally always does move at the intended velocity and we perceive it through visual feedback. However, using immersive virtual reality (IVR), it is possible to alter properties of the moving body, and give people illusory experiences of having a body with a limb that is moving differently to the real movements - though nevertheless preserving the illusion that it is your limb. Here, we present a study on possible alterations of space and body perception due to visuomotor adaptation, using the technique of embodiment in IVR. Below, we first connect our scientific questions to the past literature and then explain our methods.

The study of visuomotor adaptation to new environmental contingencies can be traced back to Helmholtz [1910]. His subjects were asked to point with their finger at targets, while wearing prisms that displaced the entire visual field to one side. This and later studies showed that large performance errors occur initially towards the directions of displacement, but then quickly decline [e.g., Baily 1972; Redding and Wallace 1993; Welch 1978, 1986]. As soon as the prisms are removed, subjects make erroneous movements in the opposite direction to the displacement. These errors are also known as after-effects of adaptation and have been suggested to occur due to proprioceptive recalibration, motor learning or even a recalibration of visual space (given that prisms displace not only the hand, but also the target and the entire workspace) [e.g., Harris 1965; Hay and Pick Jr 1966; Goodale and Milner 1992; Redding and Wallace 1996; Clower and Boussaoud 2000; Berniker and Kording 2008; Welch and Sampanes 2008; Izawa et al. 2012; Synofzik et al. 2006, 2008; Clayton et al. 2014].

In addition to the changes in motor commands, such manipulations are also thought to change the internal models of the body and the world, which predict the sensory feedback from motor commands (for a review see [Shadmehr et al. 2010]). Such perceptual recalibrations have been suggested to involve a global topological realignment, in a way that adaptation within a trained region of space generalize to other untrained regions [Bedford 1993]. In this approach, perceptual judgments of space have also been shown to be altered after visuomotor adaptation, e.g. reachability judgments [Bourgeois and Coello 2012; de Grave et al. 2011; Mon-Williams and Bingham 2007; Rodríguez-Herreros et al. 2013]. It was initially suggested that motor-related information contributes to the perception of reachable space, after it was observed that brain motor areas are involved in the perception of reachability [Coello et al. 2008]. It has been further supported by recent studies that space perception relies on motor anticipation processes, since modifying the relation between visual target distance and reach movement can affect the perceived reachable space. Bourgeois and Coello [2012] showed that spatial perception was congruently modified by the motor experience during visuomotor adaptation to 
shifted visual feedback towards or further away from the body. However, de Grave et al. [2011] reported that such modifications are not correlated with the amount of visuomotor adaptation when the visual feedback is shifted sideways. Moreover, Mon-Williams and Bingham [2007], showed that the relation between visual distance and reach distance can be modified by providing distorted haptic feedback during reaching movements, while they showed that these calibrations are not cognitively perceived.

All of the above-mentioned studies spatially modified the visual feedback. However, spatial distortions such as those induced by prism adaptation involve a constant shift that is independent of the ongoing action's kinematics. Irrespective of how fast we move we experience the same displacement, and the link with the action relies on the end-point (or final extension) of our movements. This spatial misalignment is different from the temporal misalignment (e.g. due to additional temporal delays between one's actions and the visual feedback) in that seeing the static hand will not make any difference[de la Malla et al. 2012, 2014]. A temporal discrepancy becomes apparent once the hand is moving. In this sense, a temporal discrepancy is similar to velocity-dependent distortions. For example, suppose that we increase the velocity of the visual feedback of our arm movements compared to the real velocity, then there will be a similar spatial consequence: we will reach further and instead of making a smaller displacement to hit a target we will tend to make a slower movement. However, unlike in the temporal discrepancy case, a velocity dependent distortion will provide the modified feedback immediately.

As with spatial (e.g. [Baraduc and Wolpert 2002; Ghahramani et al. 1996; Simani et al. 2007]) and temporal distortions [Cunningham et al. 2001; Franck et al. 2001; Heron et al. 2009; Kennedy et al. 2009; de la Malla et al. 2012; Stetson et al. 2006]), velocity-depended distortions can also be adapted (for visual [Rieger et al. 2005; Krakauer et al. 2000; Knoblich and Kircher 2004], and force-field perturbations [Bock and Thomas 2011; Thomas and Bock 2010; Bays et al. 2005; Goodbody and Wolpert 1998]. In this case, movement kinematics seem to alter according to the gain distortion when adapting: movements with higher gain are slower and have lower peak velocity than movements with lower gain [Rieger et al. 2005]. Moreover, conscious detection rates of a velocity-depended manipulation have been shown to be proportional to the discrepancy [Knoblich and Kircher 2004; Sutter et al. 2008].

In addition, learning a new velocity-dependent mapping when training with one target can further generalize to multiple directions and target distances, while adaptation to a new spatial mapping is more target specific for direction, suggesting that the two processes are represented differently in the brain [Krakauer et al. 2000]. However, Thomas and Bock [2010] suggested that adaptation to position and velocity-dependent visual distortions can be based on cooperative processes, since adaptation can be facilitated if one is exposed to the two distortions sequentially and if the polarity of the distortions is the same. Although mechanisms of sensorimotor adaptation to new velocities and new spatial feedback have been investigated before both separately and together, it is unknown as to whether these adaptive responses affect perceptual judgments of space differently. Our first goal was to enhance current knowledge by investigating whether spatial and spatiotemporal feedback of our movements similarly influences our perceived space as measured by judging extensions of external objects.

At the same time, when adapting to new environmental contingencies, we modify motor commands and this can change percepts of our own body and relations between our own body and external objects. Studying body perception after adapting to sersorimotor distortions could be important because the substantial changes in the motor commands to adapt to the new contingencies could lead to a modification of the way we perceive the parts of the body subserving these changed actions or even the 
sense of agency of our movements.

An early notion of Harris [1965], supported the idea that visual distortion of the perceived position of a limb with respect to its felt position (e.g. by displacing prisms) produces no alteration of the sense of ownership towards the body: the position sense is actually recalibrated to conform with the visual information. However, with displacing prism there is a constant visual displacement of the arm and a dislocated body, inevitably affecting the ecological validity of our body image.

Moreover, numerous studies have varied discrepancies between hand movements and the corresponding visual feedback such as on the spatial [Farrer et al. 2003, 2008; Fourneret and Jeannerod 1998; Franck et al. 2001; Slachevsky et al. 2001], temporal [Farrer et al. 2008; Franck et al. 2001; Leube et al. 2003; Shimada et al. 2010] or spatiotemporal [Knoblich and Kircher 2004] domain, providing evidence that such discrepancies were consciously detected only when they exceeded a certain threshold. So far, these studies use prisms, cursors or virtual hands, giving just a visual feedback of the movement ignoring the possible effect on body ownership and agency when the adapted actions are perceived through a collocated body, seen from a first person perspective.

Related to these possible effects of visuomotor adaptation on the perception of our own body, there is strong evidence that an arrangement of synchronous multiple sensory mechanisms, such as vision, touch, proprioception and motor control can result in the illusion that an object or even a full virtual body or part of it is our own. In a now classical study known as the "rubber hand illusion" (RHI), it was shown that synchronous tapping on a person's hidden real arm and an aligned visible rubber arm placed in front of them, results in a feeling of ownership of the fake arm [Botvinick and Cohen 1998]. In an earlier study, phantom limb patients viewed the reflection of the intact arm through a mirror, and when this arm was touched, they reported feeling the touch in the amputated (phantom) limb (Ramachandran et al.(1995)). IVR has more recently been used to investigate further aspects of this illusion, while providing a full-body experience, seen from a first person perspective [Llobera et al. 2013; Slater et al. 2009, 2010]. Based on such illusions, there is work showing that it is possible to have an illusory sense of ownership over a body that has a different body shape, body size or spatial position by introducing combinations of congruent sensory information [Ehrsson et al. 2005; Ehrsson 2009; Kilteni et al. 2012; Banakou et al. 2013].

Here, we make use of these findings, creating a novel setup where the body and its actions can be manipulated and still perceived as a whole. In our experiments we have created a rich stimulation of altered perceived movements consequences, by exploiting the perceptual illusion of embodiment in IVR. We immersed participants in a virtual environment and provided them with a virtual body perceived from a first person perspective with respect to the eyes of that body in order to study alterations to space and body perception after adapting to visual distorsions.

In order to explore the effects of different mechanisms of sensorimotor adaptation on space perception, we ran two experiments where we study the effects of dynamic displacements based on velocity (spatiotemporal displacements) and spatial displacements, when the virtual arm becomes owned as part of the body representation. Regarding adaptation, we assumed that participants would adapt relatively quickly to both distortions. To assess the changes in space perception, we asked participants to make judgments of the space within which they moved after adapting to scaled discrepancies, rather than measuring reachability judgments after one-way displacements. Previous studies have shown that scaled discrepancies of the visual feedback can adequately be adapted [e.g. van den Dobbelsteen 
et al. 2003]. We gradually changed the size of the discrepancy in order to explore the effects of various extensions. We specifically predicted that adaptation to larger displacements would lead to the illusion of the arm moving in a larger space.

We furthermore assessed the subjective feeling of body ownership over the virtual body and the feeling of control over the movements of the virtual hand (agency), after each adaptation stage. This way we aimed to test whether the illusion establishes regardless the discrepancies, as well as to assess the possible different effects of the discrepancies on body-ownership and agency on each of the two modalities (spatial vs. spatiotemporal). Although, we did not measure the actual limits for a conscious detection of the manipulations, we assumed that the latter comparison would give us further insights about how the two modalities affect the sensitivity for detection of changes, when acting through a whole virtual body.

\section{EXPERIMENT 1}

In this experiment we tested the effects of spatiotemporal manipulations on perceptual judgments of space and on body perception, thought embodiment in IVR. We hypothesised that participants would adapt to scaled discrepancies during a targeting task, and that this would lead participants to perceive that they are moving in an amplified space, while body-ownership and agency over the virtual body could be also affected by the manipulations.

\subsection{Materials and methods}

2.1.1 Materials. Participants were immersed in a virtual reality scenario by fitting them with a stereo NVIS nVisor SX111 head-mounted-display (HMD). This has dual SXGA displays with $76^{\circ} \mathrm{Hx} 64^{\circ} \mathrm{V}$ field of view per eye, with $50^{\circ}(66 \%)$ of overlap, totalling a wide field of view of $102^{\circ}$ horizontal and $64^{\circ}$ vertical, with a resolution of $1280 \times 1024$ per eye displayed at $60 \mathrm{~Hz}$. The latency of the video streamed into the HMD was below $100 \mathrm{~ms}$ (see Online Appendix for further details). Head tracking was performed by a 6 DOF Intersense IS-900 device. A 6 DOF tracker was placed on top of participants' right hand (see Fig. 2 A), hence the arm movements were tracked with a 12-camera optical motion capture system by Optitrack, which operate at sub-millimeter precision. A bluetooth Nintendo Wii remote device was held by the participant's right arm, as tool for the interaction with the virtual environment, using only the buttons on the device. The tracking capabilities of the Wii remote were not used.

The virtual environment was implemented using the Unity3D platform. The virtual model of the room was modeled in 3D Studio Max 2010, and we used animation-enabled models of male and female virtual bodies purchased from Rocketbox Studios.

A Simulink model in Matlab handled recordings and storage of tracking data. The data analysis was done with R [Team 2000]. Further technical characteristics can be found in the Online Appendix.

2.1.2 Participants. We recruited 16 right-handed participants ( 10 female, mean age $22.6 \pm 4.7$ years) by advertisement around the University campus. The experiment was approved by the Comissió Bioética of the University of Barcelona. All participants signed an informed consent form and were paid 5 euros for their participation.

2.1.3 Experimental Design. All participants performed a targeting motor task and experienced three extents of manipulation: congruent visuomotor correlations [1:1 (Congruent)], velocity gain as to experience amplified visual feedback, i.e. the hand reaches further away [2:1 (Amplified)], and greater 
velocity gain as to experience more amplified visual feedback [4:1 (mAmplified)]. In order to observe changes in space perception, they had to perform a space estimation task before any movements had been performed (Baseline), and after each of the three extents of manipulation.

In detail, after participants were immersed in the virtual world and before experiencing a virtual body, they were asked to make repeated estimations of width and height of an invisible box, after moving their right arm within the box area (Baseline condition) (see Section 2.1.4.4).

After this baseline judgement, participants performed a targeting task in three sessions (see Section 2.1.4.2) (Fig. 2-B). In the first session, they experienced no manipulation on the visual feedback of the movements, i.e the respective virtual arm was congruently (1:1) replicating the real arm's movements (Congruent condition). In the two following sessions, we gradually introduced two extensions (Amplified and mAmplified condition) of spatiotemporal (2:1 and 4:1) distortion of the arm movements, so that the virtual arm extended further along the movement's direction (see also video in Online Appendix). After each extent of manipulation (Congruent, Amplified, mAmplified), we hid the virtual body and participants repeated the space estimation task that was performed during the baseline condition (Figure 1). This resulted in overall four groups of space estimations for each dimension (width and height): two pre-manipulation judgments (Baseline, Congruent) and two post-manipulation judgments (Amplified, mAmplified), which allowed us to test our prediction that possible adaptation to scaled displacements would lead to the illusion of the arm moving in a larger space.

In order to test whether participants had experienced an illusion of ownership and felt control towards the virtual body before and after the distorted arm movements, a questionnaire was administered after each targeting task trial (Congruent, Amplified, mAmplified). With the purpose of testing whether participants were successfully adapting to the manipulation, performance to the targeting task was also evaluated, by measuring the number of successful hits during the target game in each trial.

\subsubsection{Procedure.}

2.1.4.1 Preparation. The participant was seated in the VR lab (Fig. 2-A). The Wii remote was given to them in order to interact with the virtual environment. An Optitrack tracker was attached to their right hand and they were advised to keep their elbow straight at all times, including during movements. After the experimenter verbally gave the instructions, the participants donned the HMD and they were immersed in the virtual room, providing them first person perspective view of a gendermatched virtual body, placed in a similar position and posture with respect to their own body (Fig. 2). The HMD was calibrated for each participant using the method described in [Grechkin et al. 2010]. Participants were instructed to describe the virtual room, in order to familiarize themselves with the environment and with the virtual body, which could be also seen as reflection in a virtual mirror.

2.1.4.2 Targeting task. In order to study the effects of visuomotor adaptation, we designed a task where participant had to move their arm, while the visual feedback of the movements was delivered through the virtual arm. The participant was asked to play a targeting game, where he/she had to shoot floating balls (Fig. 2-B). The game lasted 2.5 minutes for each manipulation extent. A semi-transparent ball appeared in a pseudo-random position around the reachable space of the participant's virtual representation (Fig. 2-C). A ball appeared every 6 seconds and remained visible (available for shooting) for 4 seconds. When the participant pressed the trigger button on the Wii remote, a red beam would appear from the virtual representation of the Wii remote and in case of collision with the target-ball, 


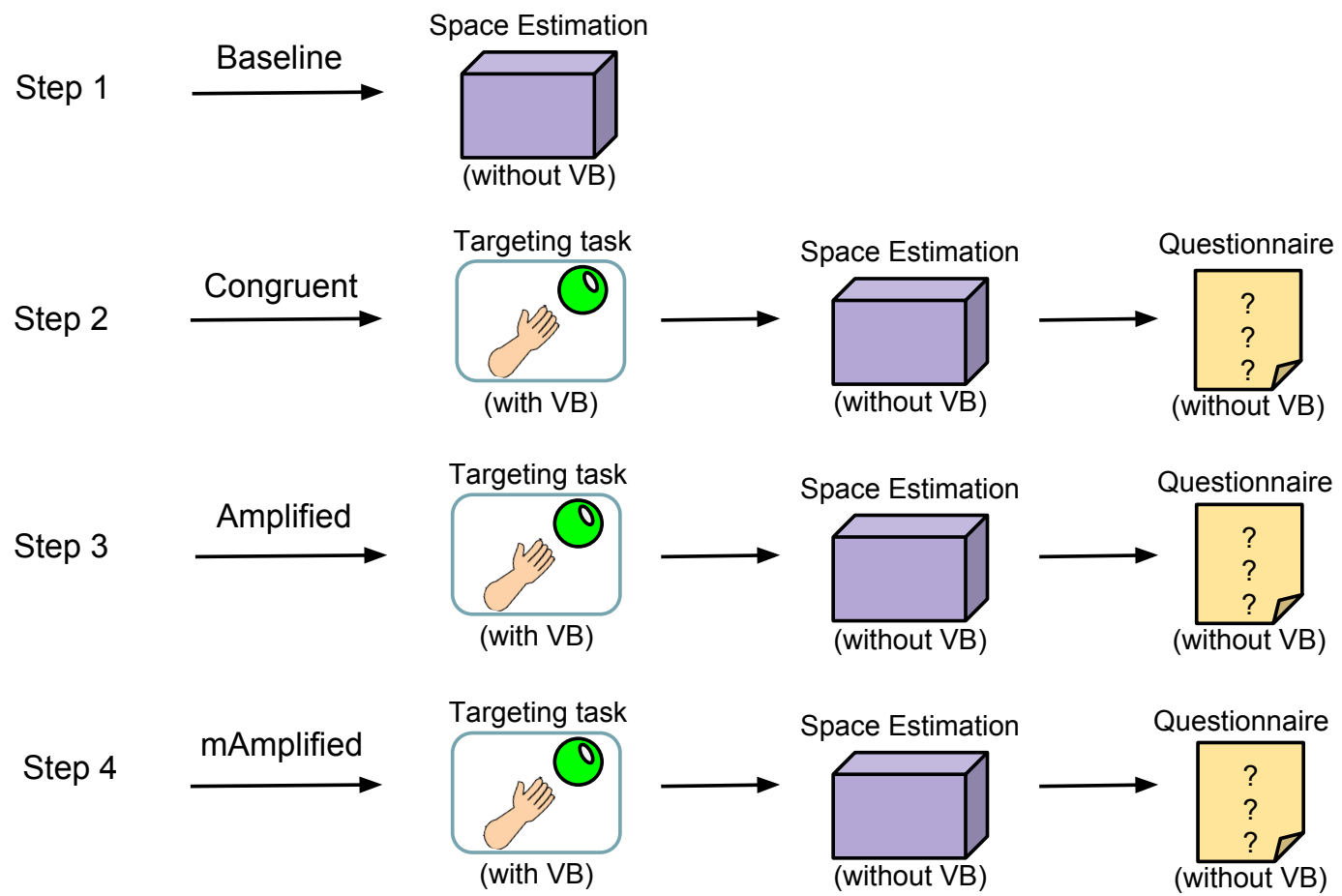

Fig. 1. Experimental procedure: All participants followed the above steps in both Experiment 1 and 2. Participants could see the virtual body (VB) during the targeting task, but not during the space estimation task or while answering the embodiment questionnaire.

the latter would turn opaque and a feedback sound would occur. We assigned greater probability for the balls to appear on the upper, left and right parts of the reachable space, rather than the center, in order to provoke larger movements and longer exposure to the manipulation conditions.

2.1.4.3 Manipulation. The spatiotemporal manipulation was achieved in two steps. First we estimated the current velocity of the arm, using the displacement of the arm from frame to frame ( $60 \mathrm{fps}$ ). To improve the estimation, we averaged the calculated velocities of four sequential frames. Then, in a second step we applied a weight [2 times faster (Amplified), 4 times faster (mAmplified)] to the estimated velocity and we applied this to compute the new position of the virtual arm.

\subsubsection{Response Variables.}

Space estimation: In order to measure changes in space perception due to the manipulations, participants are asked to perform a perceptual judgment task before and after each manipulation extent. The participant was initially immersed in the virtual room, but the virtual body was not visible, so that the perceptual judgment would be made based only on proprioception and not on the visual feedback. The participant was asked to judge the size of an invisible box that was placed around the hand (Baseline) (Fig.3. A). A collision with the box's sides would cause a vibration on the held Wii remote. Hence, 


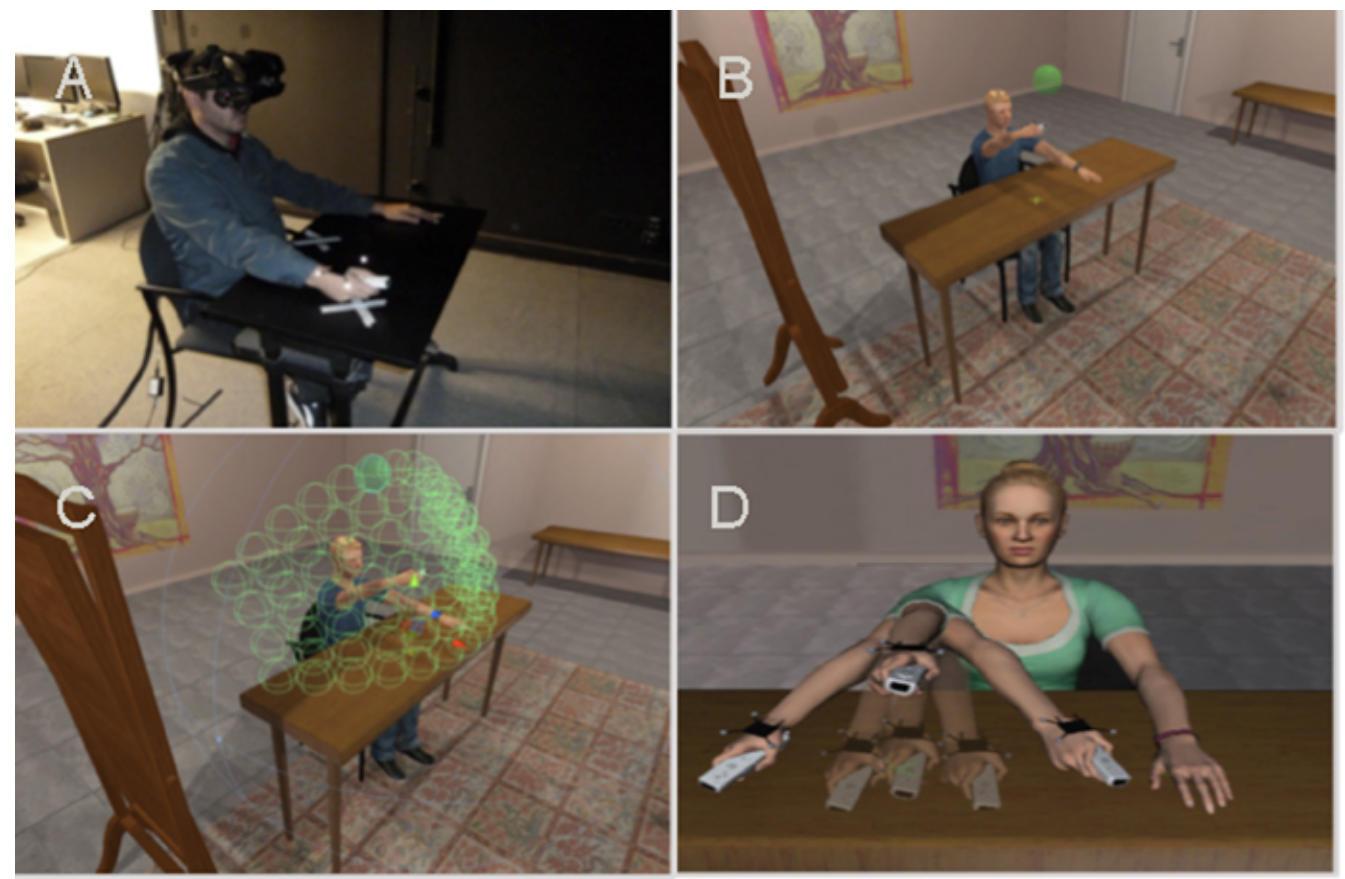

Fig. 2. (A) Participant seated in the VR lab wearing an HMD. An Optitrack tracker is attached to the right hand while with the same hand he holds a Wii remote. (B) Immersive environment. Participants can see a virtual body, seated in similar position to theirs, from a first person perspective. Target semi-transparent balls appear around avatar's reachable space. (C) The target positions were predefined and distributed on a partial sphere around the avatar's right shoulder, as to always having the same distance from the hand. (D) Amplified movements. Movements of the real hand (in transparent) are scaled towards the direction of the movement relative to the initial position, resulting amplified hand positions (in opaque).

by moving the arm upwards, downwards, towards the left and the right side, they could estimate the width and the height of the box, using only judgments based on proprioception and vibrotactile feedback. The experimenter instructed participants to "feel" the sides of the box, repeating the order of the movements three times: "up, down, right and left". The actual size of the box was width $=0.19 \mathrm{~m}$, height $=0.16 \mathrm{~m}$ and length $=0.32 \mathrm{~m}$. After sensing the sides of the box three times, they rested their arm on the table and a visible box appeared 4 times in front of them (purple box in Fig. 3-B). Using the Wii remote's cross buttons, they were able to scale this box, in order to give an estimation of the size of the previously sensed invisible one: right-left buttons adjusting for width, up and down buttons adjusting for the height. The box appeared each time in different positions in order to avoid judgments relative to previous estimates. The procedure to feel and estimate the box was repeated after each of the three trials of the targeting game (Congruent, Amplified, mAmplified): participants placed the right arm on the initial position, the virtual body turned invisible again and they performed the estimation task. With this method we measured the after-effects of the adaptation on space perception, expecting that participants would overestimate the size of the box after the adaptation to the amplified movements

Questionnaire: Since our method rests on the body ownership illusion, we needed to ensure that the illusion was established. Moreover, in order to assess the changes of self perception due to the 

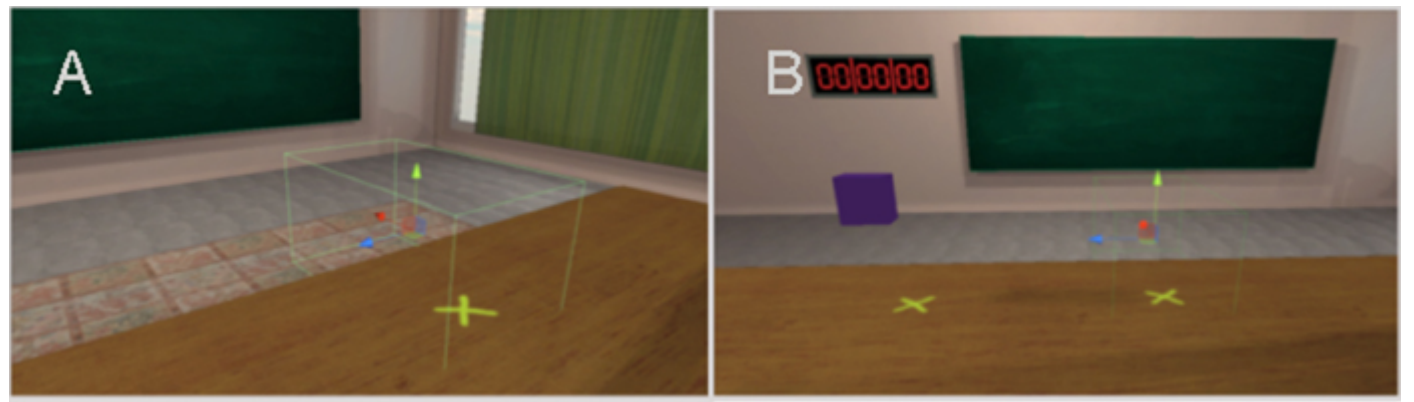

Fig. 3. (A) An invisible box was placed around the initial arm position. Vibration in the Wii remote would indicate collisions with the sides of the box. Collision with the real table would indicate the bottom side of the box. (B) The purple box appeared 4 times in different positions across the virtual table's edge. Participants could scale the box up and down using the Wii remote, until it reached the size of the "felt" invisible box. The green wired box is only for facilitating the reader. This was not visible to the participant.

manipulation, we needed to test how the illusion was affected after each extent. For this reason, we administered a questionnaire after each Space estimation (excluding Baseline), which was designed to assess the level and quality of the embodiment illusion experienced by the participants and it was based on that of Botvinick and Cohen [Botvinick and Cohen 1998]. The questionnaire appeared on a virtual blackboard inside the virtual world. Participants were asked to rate 5 statements appearing in a random order on Likert scale from 1 (totally disagree) to 7 (totally agree) (see Table III in Online Appendix). The first question 1 (Q1) referred to proprioception (self-localization); Q2 is concerned with the subjective strength of the ownership illusion and Q3 with the sense of motor control (agency). Q4 and Q5 were considered as the control questions. In a previous study, Yuan and Steed [2010] had shown that levels of ownership and agency were not affected by small spatial distortions. We were expecting similar results here, for both spatial and spatiotemporal manipulation.

Score: The number of successful hits (score) during the targeting game on each trial was also evaluated, in order to ensure that there was adaptation to the distorted visual feedback. Participants could see the score during each trial in order to keep them attentive to the task. We were expecting that due to incremental extents of manipulation, adaptation and training from previous sessions would facilitate the task.

\subsection{Results}

Data from one participant were removed from the analysis due to misunderstanding of the instructions (the participant made extremely exaggerated estimations of the box). Hence, we analyzed the data from 15 participants.

Space estimation: The actual sizes of the box were subtracted from the width and the height estimations. For each estimation trial (Baseline, Congruent, Amplified, mAmplified) we averaged the four sequential estimates and used the result as the dependent variable. Fig. 4 shows the mean overestimations at each extent of manipulation. The figure suggests differences between baseline judgments (Baseline, Congruent) and judgments after amplified extents (Amplified, mAmplified). 

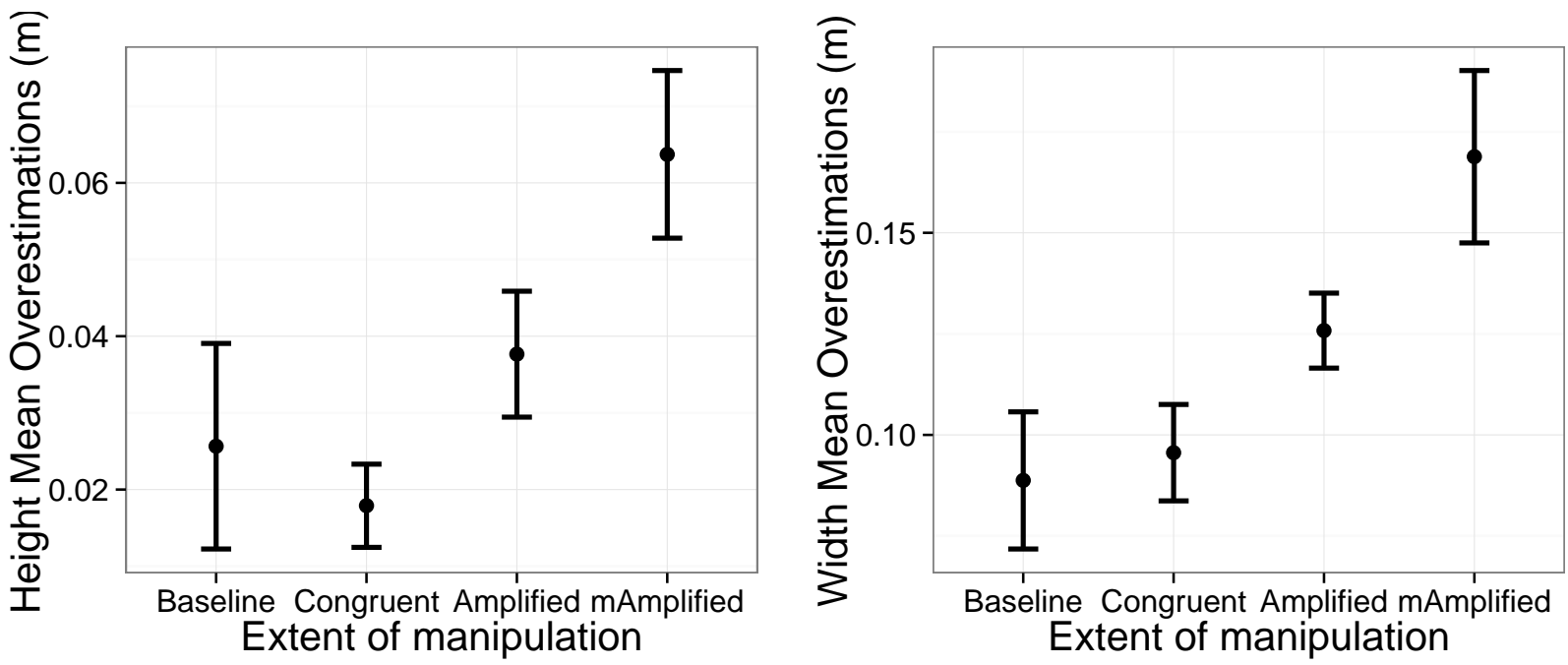

Fig. 4. Spatiotemporal manipulation. Mean and standard error bars of height (left) and width (right) space overestimations of manipulation extents Baseline, Congruent, Amplified, mAmplified. Plotting methods are handling within-subjects variable, removing inter-subjects variability. Overestimations were greater in the "width" dimension

We conducted an ANOVA with a linear mixed-effects regression (using the $R$ function lme [Pinheiro et al. 2012]) for width and height estimations separately, with manipulation extent as within-subject factor, representing fixed effects and participants representing the random effects and we found an effect on both width $(\mathrm{F}[3,42]=5.48, \mathrm{p}=0.0029)$ and height $(\mathrm{F}[3,42]=4.06, \mathrm{p}=0.01)$.

In order to further investigate the effect of the manipulation extent, we carried out multiple comparisons for parametric models on the linear mixed effects regression, using the Tukey test (with the $R$ function glht [Hothorn et al. 2008]). Width and height estimations were tested separately. Significantly greater estimations of width and height were observed on the mAmplified compared to both Baseline (width: $\mathrm{p}<0.001$, height: $\mathrm{p}=0.019$ ) and Congruent condition (width: $\mathrm{p}=0.0024$, height: $\mathrm{p}=0.003$, one-tailed, using FDR [Benjamini Hochberg] corrections). Small but not significant differences were found between Amplified and mAmplified. Moreover, it is important to report that no significance was found in the comparison between Baseline and Congruent condition (width: $p=0.98$, height: $p=0.94$, two-tailed, using FDR corrections) (see also Fig. 4 and Table I, as well as Tables IV and V in Online Appendix).

Questionnaire: The responses on the questionnaire suggested some differences on each manipulation extent (see Fig. 5, also Table VI). Here, there were only three levels of the manipulation extent factor (Congruent, Amplified, mAmplified), since the questionnaire was not administered for the baseline trial.

We treat each question as an ordered categorical variable and we used an ordered logistic regression for each question (using the $R$ function $\mathrm{clm}$ ). Levels of self-localization significantly decreased as the manipulation extent increased $\left[\chi^{2}[1]=12.681, p<0.001\right]$. Similarly, levels of agency were negatively related with the manipulation extent $\left[\chi^{2}[1]=10.79, p=0.0010\right]$. Level of manipulation had no effect on the levels of ownership $\left[\chi^{2}[1]=0.5, p=0.48\right]$. Similarly, the control question on ownership (Q5) revealed no effects of the manipulation extent $\left[\chi^{2}[1]=0.78, p=0.37\right]$. No significant effects found for the control 
Table I. Spatiotemporal manipulation. Results of post hoc analysis (using Tukey test) on linear mixed effects regression model for space estimation. Width and height estimations were tested separately. Since we expected overestimations after applying the manipulations, the comparisons are one-tailed, except for comparisons between Baseline and Congruent where we did not expect a direction on the differences. We applied FDR [Benjamini Hochberg].

\begin{tabular}{|c|c|c|}
\hline Compared Levels & Width & Height \\
\hline & p-value & p-value \\
\hline Baseline - Congruent & 0.98 & 0.94 \\
\hline Baseline - Amplified & 0.19 & 0.6 \\
\hline Baseline - mAmplified & $<0.001^{*}$ & $0.018^{*}$ \\
\hline Congruent - Amplified & 0.33 & 0.31 \\
\hline Congruent - mAmplified & $0.0024^{*}$ & $0.0032^{*}$ \\
\hline Amplified - mAmplified & 0.11 & 0.14 \\
\hline
\end{tabular}

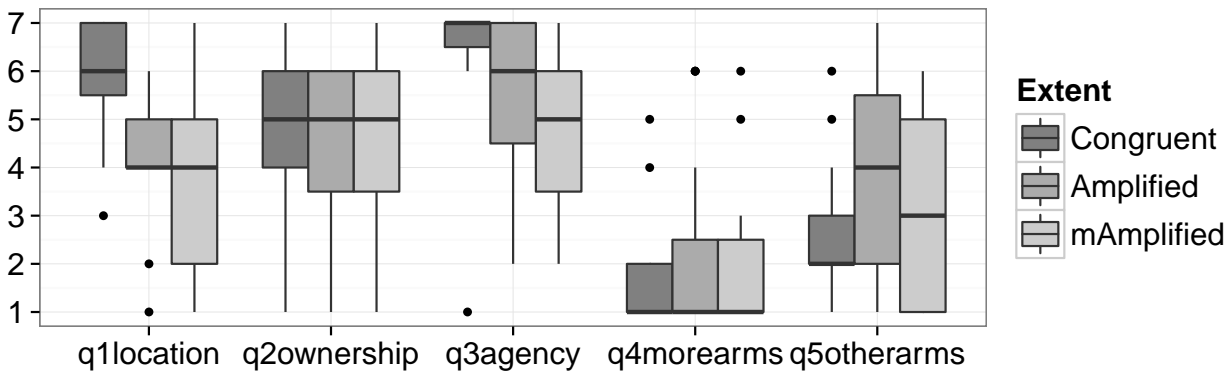

Fig. 5. Questionnaire box plots for spatiotemporal manipulations. Different shades of grey boxes indicate evaluations after each extent of manipulation (Congruent, Amplified, mAmplified). Proprioception (q1location) was significantly affected (difference of Congruent-Amplified $\mathrm{p}=0.0017$, difference of Congruent-mAmplified $\mathrm{p}<0.001$ ). Ownership levels (q2ownership) were not affected. Control question about ownership (q5otherarms) had similar results, revealing no effects of the manipulation extent. Agency levels (q3agency) were negatively correlated to the manipulation extent(difference Congruent-Amplified $\mathrm{p}=0.039$, difference Congruent-mAmplified $\mathrm{p}<0.001$ ).

question $\mathrm{Q} 4\left[\chi^{2}[1]=0.05, \mathrm{p}=0.82\right]$.

Score: Again here, there were only three levels of the manipulation extent factor (Congruent, Amplified, mAmplified), since there was not a targeting task during the baseline trial. A significant effect of manipulation extent was found on the score of the targeting task $(F[2,28]=5.32, p=0.01)$. The post hoc analysis (using Tuckey test) on the manipulation extents showed a significantly positive change of the score from the Congruent to mAmplified [Congruent-Amplified: $\mathrm{p}=0.08$ and Congruent-mAmplified: $\mathrm{p}=0.0037$, Amplified-mAmplified:p=0.53] (two-tailed, using FDR corrections). 


\subsection{Discussion}

The results suggest that spatiotemporal manipulation can significantly change participants' proprioceptive judgments of a virtual object's size as the manipulation extent increases to 2 and 4 times faster. Moreover, it seems that the manipulations do not affect the perceived body-ownership, though the perceived agency of the movements is affected, even though it does not completely vanish.

In this experiment, we introduced spatiotemporal shifts, something that has not been done in previous studies on visuomotor adaptation that involved perceptual judgments of space. We used scaled distortions, assuming that this will cause extension of the perceived space within where the arm has moved. We furthermore gave a continuous visual feedback on a 3D space, through an entire virtual body in an immersive virtual environment, rather than an endpoint feedback through a cursor on a 2D screen and we measured the subjective experience of embodiment on each level of manipulation.

Considering our results, greater overestimations were observed when visual feedback of 2 (Amplified) and 4 (mAmplified) times faster movements were introduced. This finding is interesting, since transfer of adaptation to a perceptual task has been quite controversial. Although, in the paper of de la Malla et al. [2014] this issue was meticulously discussed, mainly suggesting that the transfer of adapted delays is task specific.

In our case, we assumed that the adaptive behavior would transfer to space perception judgments, based on previous studies in visuomotor adaptation that involved perceptual judgments of space [Bourgeois and Coello 2012; de Grave et al. 2011] for spatial manipulations, and we assumed a similar behavior for spatiotemporal manipulations.

If adaptation to scaled shifts would transfer to the perceptual judgments, then overestimations of the perceived space was our expected effect. Ferrel et al. [2000] noticed that when changing the scale of the visual display individuals had the strong illusory sensation of performing movements of different amplitudes though the actual distance was kept constant. Similarly, previous studies experimentally showed adaptation to spatial distortions to alter our perception of space e.g. reachability judgments to the direction of the manipulation [Bourgeois and Coello 2012]. Here, we show first time evidence that spatiotemporal distortions to scaled shifts can also lead to amplification of the perceived space. Further studies should test whether adaptation to slower movements could cause an equivalent shrinking of the perceive space.

As expected no significant differences on the space estimations before (Baseline) and after the exposure to the virtual body (Congruent) were noticed, confirming that the possible differences between virtual and real body did not affect space estimations and hence confirming the ecological validity of the VR setup.

In order to ensure the establishment of the embodiment illusion before and after adapting to the manipulations, we used a questionnaire that has been used in previous published studies. Through the results, we could furthermore assess the effects of the discrepancies on body-ownership, self-localization and agency. Interestingly, the levels of ownership (Q2) after adapting to spatiotemporal manipulations seems to have remained intact. This is a novel finding, since previous studies that used the conventional setups could not assess the effects of the manipulations on body- ownership. This result confirms the earlier notion of Harris(1965), that supported that visual distortion of the perceived position of a limb with respect to its felt position (e.g. by displacing prisms) produces no alteration of the sense of ownership: the position sense is actually recalibrated to conform with the visual information (Harris, 1965) (see also Experiment 2). However, previous studies in body-ownership have shown that a 
completely asynchronous visuomotor correlation could easily break the illusion of ownership (Kokkinara2014). Future studies should study the limits of the visual manipulation that such an illusion can afford.

However, given the results on self-localization and agency levels (Q1 and Q3), participants seems that consciously detected the mismatches on the correlation of the virtual and the real hand, as a function of the applied extent of manipulation. Our results are comparable with those of Knoblich and Kircher [2004], where detection rate of spatiotemporal discrepancies varied as a function of the manipulation level, independently of the initial drawing velocity.

Here, we used a different targeting task than in the classical adaptation studies, that included natural movement in 3D space. Participants had no problem adapting to any level of manipulation applied, since they always managed to successfully complete the task: mean values are above 24 (successful hits) out of 26 for each case (see Online Appendix, Fig. 8). Arm movements were fully adjusted to feedback distortion, confirming that participants showed similar adapting behaviour as in the classical pointing tasks. Moreover, other studies have shown that visuomotor mappings of simply scaling size are quite easy to adapt for two directions, if rescaling applied in both directions [Bedford 1994; van den Dobbelsteen et al. 2003], which was also the case for our study (only for multiple directions). Even though the task difficulty was greater for Amplified and mAmplified due to the greater distortions, we assume that the gradual adaptation and the training on previous trials led to higher scores on those levels [Lazar and Van Laer 1968; Welch et al. 1993].

\section{EXPERIMENT 2}

In Experiment 1 we assessed adaptation to spatiotemporal manipulations, providing new insights about the subsequent effects on space and body perception. In Experiment 2, we tested the equivalent effects of adaptation to spatial manipulations. Although previous studies have provided evidence that visuomotor adaptation to spatial distortions can affect space judgements (i.e. reachability judgements), here we aimed to confirm previous results using our setup. We hypothesised that, similarly to the spatiotemporal manipulations, participants would adapt to scaled spatial discrepancies during the targeting task, and that this could lead to after-effects on space and body perception.

\subsection{Materials and methods}

Material and methods were the same as in Experiment 1. We recruited a different group of 16 righthanded participants ( 9 female, mean age $22.6 \pm 4.1$ years). All participant experienced three extents of manipulations (Congruent, Amplified and mAmplified), but instead of adding a velocity gain, this time we introduced angular drifts.

Manipulation. Similarly to Experiment 1, we used two extents of manipulation. In order to calculate equivalent standard spatial offsets to the spatiotemporal manipulation drifts, we ran a pilot study and recorded virtual and real hand positions during the 2.5 minutes of the shooting game. We then calculated the average angular offset between the real and the virtual arm for each extent of manipulation. The result was $12^{\circ}$ and $22^{\circ}$ of angular offset for the Amplified (2:1) and mAmplified (4:1) extents respectively. Hence, we added $12^{\circ}$ or $22^{\circ}$ of angular drift to the current angle of the arm. The direction of the angular offset was defined from the direction of the current position, relative to an origin vector, which lay across the right virtual arm leaned on the table in line with the shoulder (Fig. 2-D). Hence, we gave a scaled visual feedback of the arm at all times. In order to avoid "jumps" due to the changes 

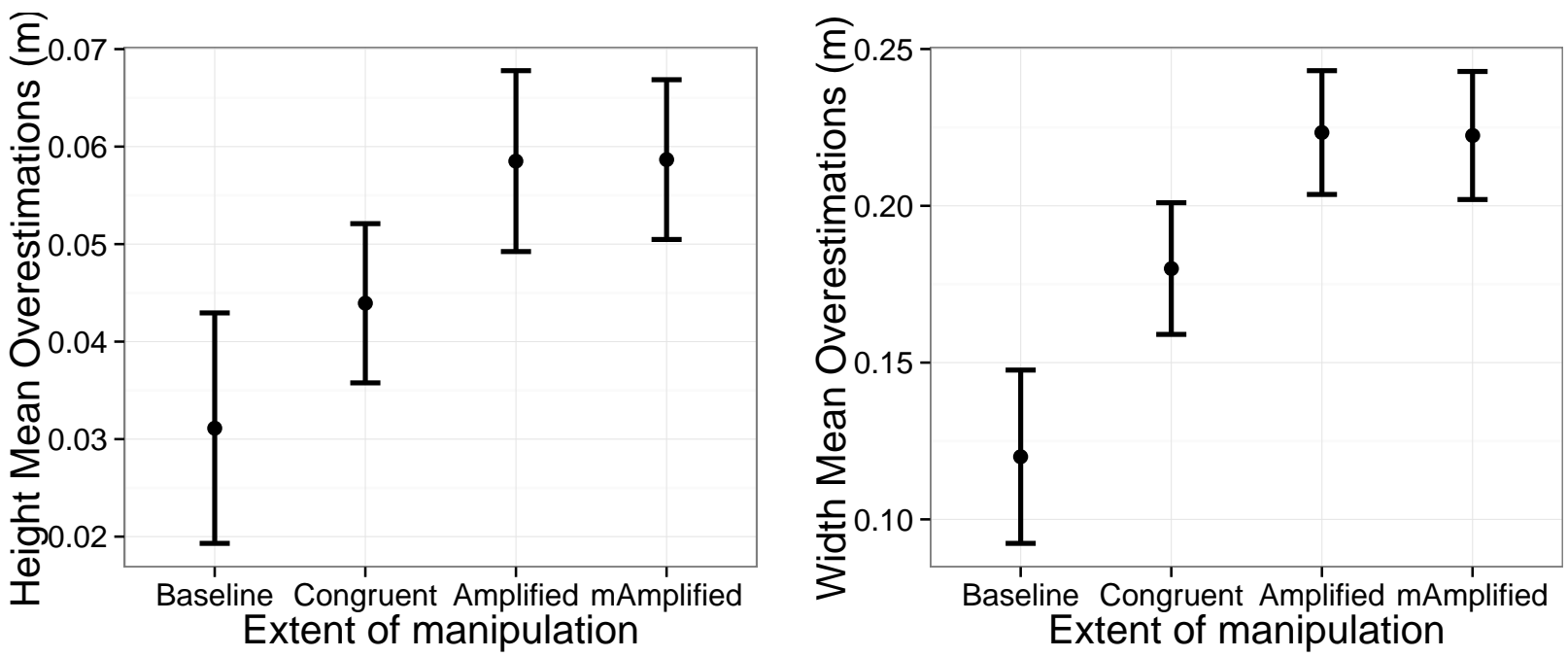

Fig. 6. Spatial manipulation. Mean and standard error bars of height (left) and width (right) space overestimations of manipulation extents Baseline, Congruent, Amplified, mAmplified. Plotting methods are handling within-subjects variable, removing inter-subjects variability. Overestimations were greater in the "width" dimension

of direction (relative to the origin) or "floating" of the arm on starting position, we created a virtual sphere around the initial position and we instructed participants to avoid touching it during the game. To enhance this instruction, a disturbing noise and a vibration through the Wii remote were delivered by the system whenever their arm was colliding with this virtual sphere.

The experimental procedure was the same as in Experiment 1 (Figure 1) and we used the same response variables.

\subsection{Results}

Space estimation: We conducted the same type of analysis as in experiment 1 . The ANOVA on a linear mixed-effects regression showed an effect of manipulations extent on width $(\mathrm{F}[3,45]=4.72$, $\mathrm{p}<0.001)$, but not on height $(\mathrm{F}[3,45]=1.95, \mathrm{p}=0.13)$ overestimations.

The post-hoc analysis showed significantly greater estimations of width on Amplified and mAmplified compared to the Baseline for width (Amplified-Baseline: $p=0.0031$, mAmplified-Baseline: $p=0.0033$ ), but comparisons for height did not reach significance (Amplified-Baseline: $\mathrm{p}=0.09$, mAmplified-Baseline: $\mathrm{p}=0.09$ ). No differences can be seen between Amplified and mAmplified.

No significant differences were found on the comparison between Baseline and Congruent condition (width : $\mathrm{p}=0.23$, height: $\mathrm{p}=0.77$, two-tailed, using FDR corrections) (see also Fig. 6 and Table II, as well as Tables IV and V in Online Appendix).

Questionnaire: The responses on the questionnaire suggested no differences between the three extents of manipulation for the spatial condition, whereas some differences can be seen on the spatiotemporal condition (see Fig. 7, also Table VI). The questionnaire was administered only after three levels of the manipulation extent (Congruent, Amplified, mAmplified).

The ordered logistic regression showed no relationship was found between the manipulation extent variable and the levels of self-localization $\left[\chi^{2}[1]=1.2, p=0.27\right]$, the levels of agency $\left[\chi^{2}[1]=2.56, p=0.11\right]$, the levels of ownership $\left[\chi^{2}[1]=0.68, p=0.4\right]$, the control question on ownership (Q5) $\left[\chi^{2}[1]=0.21, p=0.64\right]$ 
Table II. Spatial manipulation. Results of post hoc analysis (using Tukey test) on linear mixed effects regression model for space estimation. Width and height estimations were tested separately.

Comparisons are one-tailed, except for comparisons between Baseline and

Congruent. We applied FDR [Benjamini Hochberg]

\begin{tabular}{|c|c|c|}
\hline Compared Levels & Width & Height \\
\hline & p-value & p-value \\
\hline Baseline - Congruent & 0.23 & 0.77 \\
\hline Baseline - Amplified & $0.0031^{*}$ & 0.097 \\
\hline Baseline - mAmplified & $0.0033^{*}$ & 0.095 \\
\hline Congruent - Amplified & 0.33 & 0.47 \\
\hline Congruent - mAmplified & 0.34 & 0.47 \\
\hline Amplified - mAmplified & 0.96 & 0.95 \\
\hline
\end{tabular}

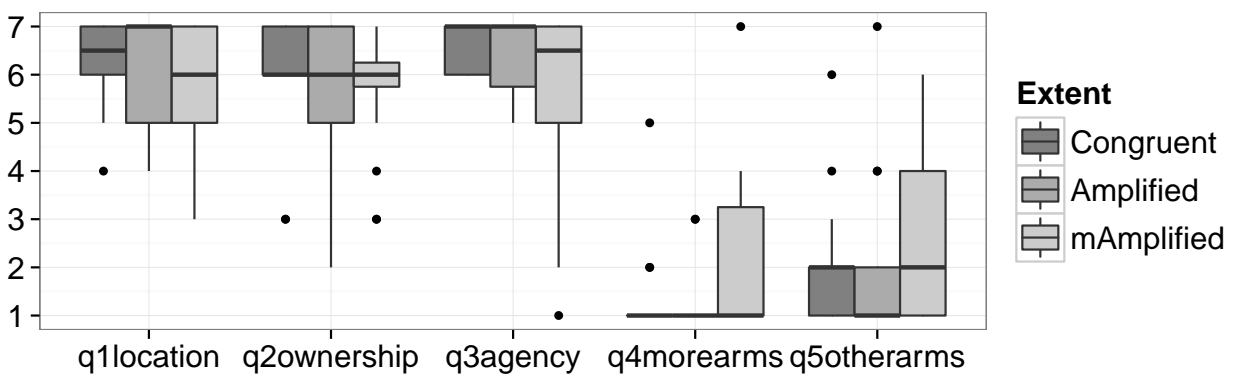

Fig. 7. Questionnaire box plots for spatial manipulations. Different shades of grey boxes indicate evaluations after each extent of manipulation (Congruent, Amplified, mAmplified). Non of the questions was affected by the manipulation effect. However, levels of ownership were higher than after spatial than after spatiotemporal manipulations (q2ownership: $p<0.001$, q5otherarms: $\mathrm{p}=0.004$ )

or the control question $\mathrm{Q} 4\left[\chi^{2}[1]=3.34, \mathrm{p}=0.067\right]$.

Score: No significant effect of extent of manipulation was found on the score of the targeting task $(\mathrm{F}[2,30]=2.4, \mathrm{p}=0.108)$. The post hoc analysis (using Tuckey test) on the manipulation extents showed no differences [Congruent-Amplified: $\mathrm{p}=0.1$ and Congruent-mAmplified: $\mathrm{p}=0.2$, Amplified-mAmplified: $\mathrm{p}=0.95]$ (two-tailed, using FDR corrections).

Comparison with spatiotemporal manipulation: We conducted an ANOVA on a linear mixed-effects regression for width and height estimations separately, with manipulation extent as within-subject factor and manipulation type (spatial-spatiotemporal) as between-subject factor, representing fixed effects. We found no effect of the manipulation type (width: $F[3,29]=1.86, p=0.18$, height: $F[3,29]=0.24$, $\mathrm{p}=0.63$ ). No significant interaction was found between manipulation type and manipulation extent 
(width: $F[3,87]=1.75, p=0.32$, height: $F[3,87]=1.076, p=0.36$ ).

Comparing questionnaire data for manipulation type and manipulations extents with an ordered logistic regression for each question, We found that levels of ownership were greater in spatial than in spatiotemporal $(\mathrm{p}<0.001)\left[\chi^{2}[1]=15.6184, \mathrm{p}<0.00001\right]$, while the control question on ownership (Q5) responses were lower in spatial than in spatiotemporal $(p=0.00476)\left[\chi^{2}[1]=8.2170, p=0.00415\right]$. We found no interaction between the two factors for any of the questions.

A significant effect of both manipulation type and extent of manipulation was found on the score of the targeting task $(\mathrm{F}[1,29]=9.19, \mathrm{p}=0.005$ and $\mathrm{F}[2,58]=6.34, \mathrm{p}=0.003$ respectively $)$

\subsection{Discussion}

Similarly to spatiotemporal manipulations, spatial manipulations of $12^{\circ}$ (Amplified) and $22^{\circ}$ (mAmplified) degrees of angular offset had en effect on the perceived virtual object's size, but significant only for the horizontal dimension (width). Moreover, while the mismatched information did not affect the sense of body-ownership, opposite to Experiment 1, the perceived arm location and agency of the movement were not affected in this case.

The results confirm previous studies that showed adaptation to spatial distortion to affect perceptual judgments of space, e.g. reachability judgments [Bourgeois and Coello 2012; de Grave et al. 2011]. Moreover, we enhance previous results, showing that the effect is not limited to single directions, but perceptual judgments of 3D space can be altered after adaptation to scaled distortion. However, although changes in height estimation were observed, these were not significant. A possible reason could be that participants were not moving as much in the vertical dimension in front of them, due to the "no touch" sphere that was placed around the initial position, hence adaptation might have not been complete.

In the spatial case overestimations did not seem to gradually increase with larger amplifications, although the final amount of overestimation in mAmplified was similar to both spatial and spatiotemporal experiments. A possible explanation could be that there is a limit of overestimations of the perceived space, whichever adaptation method is used. Considering this assumption, adaptation to spatial manipulation leaded to reaching of this limit faster, whereas smaller steps were needed for spatiotemporal manipulation. Moreover, although we see, as we expected, adaptation in both, this could be of a different kind. In the spatial manipulation subjects might be shifting spatial components of the motor command (aiming further/closer), while in the spatiotemporal, the adaptation might be based more in anticipation (more emphasis on online information). Since online information is missing during the perceptual judgment, aftereffects might decline faster.

Similar to Experiment 1, no significant differences on the space estimations before (Baseline) and after the exposure to the virtual body (Congruent) were noticed. Mean overestimations in the Congruent condition were higher after spatial manipulations than in spatiotemporal (Fig. 4 and 6, Table IV and $\mathrm{V}$ in Online material). This could be attributed to the slightly different design that included the "no touch" sphere in the spatial condition. Participants had to follow somewhat different movement patterns to reach the targets, in order to avoid the penalized zone. Although we were more interested in observing the overall existence of the effects after the manipulations, rather than a direct one-to-one comparison of each extent, we believe that further studies could study the comparison of the two in 
detail by including, for example, a "no touch" sphere in both types of manipulation.

Spatial manipulation seems to have no impact on the high levels of body-ownership (Q2), and opposite to spatiotemporal manipulations from Experiment 1, self-localization are agency (Q1, Q3) were not affected either, even with 220 of deviation. Previous studies have shown much lower detection thresholds of the manipulation (related to agency) for healthy participants (thresholds around $15^{\circ}$ ) [Farrer et al. 2008, 2003; Franck et al. 2001; Posada et al. 2007; Synofzik et al. 2006]. It is possible that the difference here is observed due to the full-body ownership illusion and the fact that the visual feedback of the manipulation derives from an arm that is directly connected to the seen body.

We observed overall higher levels of ownership after spatial manipulation than after spatiotemporal. A possible explanation could be that this occurred due to the vibrotactile feedback that was provided when participants were touching the "no touch" sphere that was placed around the initial position. Although, a contact of the moving arm with the sphere was rarely noticed, the vibration caused through the Wii remote provided a synchronous visuotactile feedback on the stimulated limb. As it has been shown in previous studies, visuotactile feedback can enhance the sense of ownership towards the virtual arm [Slater et al. 2008].

As in Experiment 1, participants adapted to any level of manipulation applied although mean scores were somewhat lower in spatial than spatiotemporal manipulations.

\section{GENERAL DISCUSSION}

In this study we showed that spatiotemporal manipulation of 2 and 4 times faster can significantly change participants' proprioceptive judgments of space, without affecting the perceived body-ownership, though affecting agency of the movements. The equivalent spatial manipulations of $11^{\circ}$ and $22^{\circ}$ of angular offset also had a significant effect on the perceived virtual object's size, however the mismatched information did not affect either the sense of body-ownership or agency.

Although both manipulations might require similar motor adjustments, and adaptation to position and velocity-dependent visual distortions have been thought to be based on cooperative processes [Bock 2003; Thomas and Bock 2010], they are also suggested to obey different rules of generalization [Krakauer et al. 2000] and it is not clear exactly how much information is shared between the two processes. Interestingly, here we noticed that spatiotemporal manipulations could more easily be detected, although they led to smaller overestimations in absolute terms. Maintaining the assumption described earlier, that the two sensorimotor manipulations adapt distinguishable elements of the participants control system, the spatial should involve more spatial strategies (e.g. aiming at a different endpoint in space), while the spatiotemporal could encourage more online control and monitoring, based on actual velocity. The latter could explain the fast concious detections of the manipulation in velocity. Future studies on this field, could use this setup in order to provide further insights about what specifically is being adapted, when a whole virtual body is substituting the real one.

de Grave et al. [2011] reported that when the visual feedback is shifted sideways then modifications on perceived reachability were not always towards the same direction, concluding that judgments of reachability are largely independent of visuomotor adaptions. Using a different paradigm, here, we applied offsets towards multiple directions as to amplify the movements, and we show that the shifts alter perceptual judgments towards the scaling direction. This is in-line with the findings of Krakauer et al. [2000], who showed that adaptation to spatial distortions generalizes easier to other directions and extents, when participants already trained in multiple directions instead of a single one. Moreover, 
our findings might be an outcome of the scaled shifts applied on a 3D space with an egocentric frame of reference, along with the fact that participants could continuously perceive a visual feedback of their moving arm.

Unlike previous studies that have assessed the effects of motor adaptation in space perception, here we were interested on providing an online continuous visual feedback of the exposure to the distortion, rather than just feedback on the endpoint of each movement. This technique has been considered able to alter the correction mechanisms [Redding and Wallace 1996; Choe and Welch 1974], because it provides continuous cues about the frames of reference (e.g., linked to eye, head, shoulder) that could be involved. Results from previous studies show that endpoints of natural arm movements towards visual targets were not affected by changes in the starting position of the hand, suggesting that such movements are planned in terms of the final egocentric position [Vetter et al. 1999; Hay et al. 1971; van den Dobbelsteen et al. 2001; Polit and Bizzi 1979]. Moreover, it has been suggested that adapting through visual feedback of the hand (prism adaptation) may cause greater perceptual recalibration, rather than simple changes in motor commands, when compared to a representational feedback through a cursor [Clower and Boussaoud 2000].

As previous studies that used more simplistic virtual reality techniques, such as cursor [e.g., Krakauer et al. 2000] or virtual hands [e.g., Franck et al. 2001], we were able to study whether visuomotor adaptation leads to changes in felt hand position, avoiding the visual recalibration of the entire scene occurred in prism adaptation. However, with previous setups it was not possible to study the effect of visuomotor adaptation on body perception, since the body was either dislocated by prisms or remained hidden replacing just the hand by a cursor or a virtual hand.

Previous body-centered approaches have tried to assess the importance of a visual feedback of the limb position during action on a visuomotor adaptation task (e.g. [Desmurget et al. 1997, 1995; Elliott et al. 1991; Redding and Wallace 1996; Rossetti et al. 1994]. In this context, it has been suggested that when visual information about the target's location is integrated with kinesthetic information about the position and movements of the hand, people adapt much more readily to distortions of visual feedback that correspond to transformations with respect to the body than to distortions that are defined with respect to the world [van den Dobbelsteen et al. 2003; Vetter et al. 1999]. Here, not only we used distortions with respect to the body (shoulder), but we also enhance the input coming from the kinesthetic information about the position and the movements of the hand, with visual feedback of the entire 'own' hand and body. However, further studies will be needed, in order to prove whether participants can more readily adapt to transformations through this setup, by meticulously examining, for example, the adaptive behavior when an entire virtual body is provided versus when is not.

This setup could give also important insights about how body image can moderate our movements and the perceived space [Banakou et al. 2013], since as with the use of tools, the body image could be fairly easily altered, by manipulating the virtual body [Kilteni et al. 2012; Banakou et al. 2013] .

\section{CONCLUSIONS}

Visuomotor adaptation to spatial and spatiotemporal scaled manipulations can both change space perception. However, it is possible that the mechanisms used for the adaptation during spatiotemporal distortions lead to a faster conscious perception of manipulation, suggesting adaptation of a different kind, although body ownership remained intact in both cases. While assessing the effects of visuomotor adaptation to space and body perception, we have presented a novel paradigm where the body and 
its actions can be manipulated and perceived as a whole in an immersive virtual environment, providing insights for future studies that could use this ecologically valid setup. Unconscious adaptation to visually modified movements could be of use in future VR applications, since the field is predicted to be more and more conspicuous in the future. For example, one might be able to act in an extended virtual space, while being on a confined physical space, simply by adapting to amplified movements. The results of this research could be useful for future studies from low-level cognitive science studies to more practical applications that use virtual reality.

\section{ACKNOWLEDGEMENTS}

This research was funded by the FP7 EU VR-HYPERSPACE (AAT-285681) project funded under the Aeronautics and Air Transport (AAT) workprogramme.

\section{REFERENCES}

BAILY, J. S. 1972. Adaptation to prisms: Do proprioceptive changes mediate adapted behaviour with ballistic arm movements? The Quarterly Journal of Experimental Psychology 24, 1, 8-20.

BANAKou, D., Groten, R., AND Slater, M. 2013. Illusory ownership of a virtual child body causes overestimation of object sizes and implicit attitude changes. Proceedings of the National Academy of Sciences 110, 31, 12846-12851.

BARAduc, P. AND Wolpert, D. M. 2002. Adaptation to a visuomotor shift depends on the starting posture. Journal of neurophysiology 88, 2, 973-81.

Bays, P. M., FlanaGan, J. R., AND WOLPERT, D. M. 2005. Interference between velocity-dependent and position-dependent force-fields indicates that tasks depending on different kinematic parameters compete for motor working memory. Experimental brain research 163, 3, 400-405.

BEDFoRD, F. L. 1993. Perceptual and cognitive spatial learning. Journal of Experimental Psychology: Human Perception and Performance 19, 3, 517.

Bedford, F. L. 1994. Of computer mice and men. Cah. Psychol. Cognit 13, 405-426.

BERNiKer, M. AND KoRDing, K. 2008. Estimating the sources of motor errors for adaptation and generalization. Nature neuroscience 11, 12, 1454-1461.

Bock, O. 2003. Sensorimotor adaptation to visual distortions with different kinematic coupling. $E x$ perimental brain research. Experimentelle Hirnforschung. Expérimentation cérébrale 151, 4, 557-60.

Bock, O. AND Thomas, M. 2011. Proprioception plays a different role for sensorimotor adaptation to different distortions. Human movement science 30, 3, 415-423.

Botvinick, M. And Cohen, J. 1998. Rubber hands 'feel' touch that eyes see. Nature 391, 6669, 756.

BOURGEOIS, J. AND COELLO, Y. 2012. Effect of visuomotor calibration and uncertainty on the perception of peripersonal space. Attention, Perception, \& Psychophysics 74, 6, 1268-1283.

Choe, C. S. AND WeLCH, R. B. 1974. Variables affecting the intermanual transfer and decay of prism adaptation. Journal of experimental psychology 102, 6, 1076.

Clayton, H. A., Cressman, E. K., And Henriques, D. Y. 2014. The effect of visuomotor adaptation on proprioceptive localization: the contributions of perceptual and motor changes. Experimental brain research, 1-14.

Clower, D. M. AND Boussaoud, D. 2000. Selective use of perceptual recalibration versus visuomotor skill acquisition. Journal of Neurophysiology 84, 5, 2703-2708. 
Coello, Y., Bartolo, A., Amiri, B., Devanne, H., Houdayer, E., And Derambure, P. 2008. Perceiving what is reachable depends on motor representations: evidence from a transcranial magnetic stimulation study. PLoS One 3, 8, e2862.

Cunningham, D. W., Billock, V. A., And Tsou, B. H. 2001. Sensorimotor adaptation to violations of temporal contiguity. Psychological Science 12, 6, 532-535.

DE Grave, D. D. J., Brenner, E., And Smeets, J. B. J. 2011. Judgments of reachability are independent of visuomotor adaptation. Perception 40, 8, 962-974.

De la Malla, C., López-Moliner, J., And Brenner, E. 2012. Seeing the last part of a hitting movement is enough to adapt to a temporal delay. Journal of Vision 12, 10.

DE la MAlla, C., López-Moliner, J., AND BREnner, E. 2014. Dealing with delays does not transfer across sensorimotor tasks. Journal of vision 14, $12,8$.

de la Peña, N., Weil, P., Llobera, J., Giannopoulos, E., Pomés, A., Spanlang, B., Friedman, D., SANCHEZ-VIVES, M. V., AND SLATER, M. 2010. Immersive journalism: immersive virtual reality for the first-person experience of news. Presence: Teleoperators and Virtual Environments 19, 4, 291301.

Desmurget, M., Rossetti, Y., Jordan, M., Meckler, C., And Prablanc, C. 1997. Viewing the hand prior to movement improves accuracy of pointing performed toward the unseen contralateral hand. Experimental Brain Research 115, 1, 180-186.

Desmurget, M., Rossetti, Y., Prablanc, C., Jeannerod, M., And Stelmach, G. E. 1995. Representation of hand position prior to movement and motor variability. Canadian journal of physiology and pharmacology 73, 2, 262-272.

EHRSSON, H. H. 2009. How many arms make a pair? Perceptual illusion of having an additional limb. Perception 38, 2, 310-312.

Ehrsson, H. H., Kito, T., Sadato, N., Passingham, R. E., And Naito, E. 2005. Neural Substrate of Body Size: Illusory Feeling of Shrinking of the Waist. PLoS Biology 3, 12, e412.

Elliott, D., Garson, R. G., Goodman, D., and Chua, R. 1991. Discrete vs. continuous visual control of manual aiming. Human Movement Science 10, 4, 393-418.

Farrer, C., Bouchereau, M., Jeannerod, M., AND Franck, N. 2008. Effect of distorted visual feedback on the sense of agency. Behavioural neurology 19, 1-2, 53-7.

Farrer, C., Franck, N., GeorgiefF, N., Frith, C., Decety, J., and Jeannerod, M. 2003. Modulating the experience of agency: a positron emission tomography study. NeuroImage 18, 2, 324-333.

Ferrel, C., Leifflen, D., Orliaguet, J.-P., ANd Coello, Y. 2000. Pointing movement visually controlled through a video display: adaptation to scale change. Ergonomics 43, 4, 461-473.

FOURNERET, P. AND JEANNEROD, M. 1998. Limited conscious monitoring of motor performance in normal subjects. Neuropsychologia 36, 11, 1133-40.

Franck, N., Farrer, C., Georgieff, N., Marie-Cardine, M., Daléry, J., D’Amato, T., and JEANNEROD, M. 2001. Defective recognition of one's own actions in patients with schizophrenia. The American Journal of Psychiatry 158, 3, 454-459.

Ghahramani, Z., WolPert, D. M., AND JORDAN, M. I. 1996. Generalization to local remappings of the visuomotor coordinate transformation. The Journal of neuroscience : the official journal of the Society for Neuroscience 16, 21, 7085-96.

Goodale, M. A. AND Milner, A. D. 1992. Separate visual pathways for perception and action. Trends in neurosciences 15, 1, 20-25. 
Goodbody, S. J. AND WolpeRT, D. M. 1998. Temporal and amplitude generalization in motor learning. Journal of Neurophysiology 79, 4, 1825-1838.

Grechion, T. Y., Nguyen, T. D., Plumert, J. M., Cremer, J. F., And Kearney, J. K. 2010. How does presentation method and measurement protocol affect distance estimation in real and virtual environments? ACM Transactions on Applied Perception (TAP) 7, 4, 26.

HARRIS, C. S. 1965. Perceptual adaptation to inverted, reversed, and displaced vision. Psychological review $72,6,419$.

HAY, J. C., LANGDON, B., AND PICK, H. L. 1971. Spatial parameters of eye-hand adaptation to optical distortion. Journal of experimental psychology 91, 1, 11.

HAY, J. C. AND PICK JR, H. L. 1966. Gaze-contingent prism adaptation: optical and motor factors. Journal of experimental psychology 72, 5, 640 .

Helmholtz, H. v. 1910. Helmholtz's treatise on physiological optics. vol. iii. translated from the 3rd german edition and edited by jpc southall.

HERON, J., HANSON, J. V. M., AND WHITAKER, D. 2009. Effect before cause: supramodal recalibration of sensorimotor timing. PloS one 4, 11, e7681.

Hothorn, T., Bretz, F., AND Westfall, P. 2008. Simultaneous inference in general parametric models. Biometrical Journal 50, 3, 346-363.

Izawa, J., Criscimagna-Hemminger, S. E., AND Shadmehr, R. 2012. Cerebellar contributions to reach adaptation and learning sensory consequences of action. The Journal of Neuroscience 32, 12, $4230-4239$.

Kennedy, J. S., Buehner, M. J., And Rushton, S. K. 2009. Adaptation to sensory-motor temporal misalignment: instrumental or perceptual learning? Quarterly journal of experimental psychology (2006) 62, 3, 453-69.

Kilteni, K., Normand, J.-M., Sanchez-Vives, M. V., and Slater, M. 2012. Extending Body Space in Immersive Virtual Reality: A Very Long Arm Illusion. PLoS ONE 7, 7, e40867.

KNOBLICH, G. AND KIRCher, T. T. J. 2004. Deceiving oneself about being in control: conscious detection of changes in visuomotor coupling. Journal of experimental psychology. Human perception and performance 30, 4, 657-66.

Krakauer, J. W., Pine, Z. M., Ghilardi, M. F., AND Ghez, C. 2000. Learning of visuomotor transformations for vectorial planning of reaching trajectories. The Journal of neuroscience : the official journal of the Society for Neuroscience 20, 23, 8916-24.

LAZAR, G. AND VAN LAER, J. 1968. Adaptation to displaced vision after experience with lesser displacements. Perceptual and motor skills 26, 2, 579-582.

Leube, D., Knoblich, G., Erb.Michael, Wolfgang, G., Bartels, M., And Kircher, T. T. J. 2003. The neural correlates of perceiving one's own movements. NeuroImage 20, 4, 2084-2090.

Llobera, J., SAnchez-Vives, M., AND Slater, M. 2013. The relationship between virtual body ownership and temperature sensitivity. Journal of The Royal Society Interface 10, 85.

Mon-Williams, M. AND Bingham, G. P. 2007. Calibrating reach distance to visual targets. Journal of experimental psychology. Human perception and performance 33, 3, 645-56.

Pinheiro, J., Bates, D., DebRoy, S., SARkar, D., AND R Core Team. 2012. nlme: Linear and Nonlinear Mixed Effects Models. R package version 3.1-104.

Polit, A. AND BIZZI, E. 1979. Characteristics of motor programs underlying arm movements in monkeys. J Neurophysiol 42, 1, 183-194. 
Posada, A., Franck, N., Augier, S., Georgieff, N., And Jeannerod, M. 2007. Altered processing of sensorimotor feedback in schizophrenia. Comptes rendus biologies 330, 5, 382-8.

Redding, G. M. AND Wallace, B. 1993. Adaptive coordination and alignment of eye and hand. Journal of Motor Behavior 25, 2, 75-88.

REDDING, G. M. AND WALLACE, B. 1996. Adaptive spatial alignment and strategic perceptual-motor control. Journal of experimental psychology. Human perception and performance 22, 2, 379-94.

Rieger, M., KNOBlich, G., AND PRINZ, W. 2005. Compensation for and adaptation to changes in the environment. Experimental Brain Research 163, 4, 487-502.

RodríGuez-Herreros, B., De Grave, D. D., López-Moliner, J., Brenner, E., And Smeets, J. B. 2013. Shifted visual feedback of the hand affects reachability judgments in interception. Vision research 88, 30-37.

Rossetti, Y., Stelmach, G., Desmurget, M., Prablanc, C., And Jeannerod, M. 1994. The effect of viewing the static hand prior to movement onset on pointing kinematics and variability. Experimental Brain Research 101, 2, 323-330.

Shadmehr, R., Smith, M. A., AND Krakauer, J. W. 2010. Error correction, sensory prediction, and adaptation in motor control. Annual review of neuroscience 33, 89-108.

SHIMADA, S., QI, Y., AND HIRAKI, K. 2010. Detection of visual feedback delay in active and passive self-body movements. Experimental brain research. Experimentelle Hirnforschung. Expérimentation cérébrale 201, 2, 359-64.

Simani, M. C., MCGuire, L. M., AND SABES, P. N. 2007. Visual-shift adaptation is composed of separable sensory and task-dependent effects. Journal of neurophysiology 98, 5, 2827-2841.

Slachevsky, A., Pillon, B., Fourneret, P., Pradat-Diehl, P., Jeannerod, M., and Dubois, B. 2001. Preserved adjustment but impaired awareness in a sensory-motor conflict following prefrontal lesions. Journal of cognitive neuroscience 13, 3, 332-340.

Slater, M., Perez-Marcos, D., Ehrsson, H. H., And Sanchez-Vives, M. V. 2008. Towards a digital body: the virtual arm illusion. Frontiers in human neuroscience 2, August, 6.

Slater, M., Perez-Marcos, D., Ehrsson, H. H., And Sanchez-Vives, M. V. 2009. Inducing illusory ownership of a virtual body. Frontiers in neuroscience 3, 2, 214-20.

Slater, M., Spanlang, B., Sanchez-Vives, M. V., AND Blanke, O. 2010. First person experience of body transfer in virtual reality. PLoS ONE 5, 5, e10564.

Stetson, C., Cui, X., Montague, P. R., And Eagleman, D. M. 2006. Motor-sensory recalibration leads to an illusory reversal of action and sensation. Neuron 51, 5, 651-9.

Sutter, C., Müsseler, J., Bardos, L., Ballagas, R., AND Borchers, J. 2008. The impact of gain change on perceiving one's own actions. In Mensch \& computer. 147-156.

SYNOFZIK, M., THIER, P., AND LiNDNER, A. 2006. Internalizing agency of self-action: perception of one's own hand movements depends on an adaptable prediction about the sensory action outcome. Journal of Neurophysiology 96, 3, 1592-1601.

SynofziK, M., VosgeraU, G., AND Newen, A. 2008. Beyond the comparator model: a multifactorial two-step account of agency. Consciousness and cognition 17, 1, 219-239.

TEAM, R. C. 2000. R language definition.

Thomas, M. AND Bock, O. 2010. Is sensorimotor adaptation to position- and velocity-dependent visual distortions based on distinct adaptive processes? Human movement science 29, 2, 179-86. 
van den Dobbelsteen, J., Brenner, E., And Smeets, J. B. J. 2003. Adaptation of movement endpoints to perturbations of visual feedback. Experimental brain research. Experimentelle Hirnforschung. Expérimentation cérébrale 148, 4, 471-81.

VAN Den Dobbelsteen, J. J., Brenner, E., AND Smeets, J. B. 2001. Endpoints of arm movements to visual targets. Experimental brain research 138, 3, 279-287.

VetTer, P., Goodbody, S. J., AND Wolpert, D. M. 1999. Evidence for an eye-centered spherical representation of the visuomotor map. Journal of Neurophysiology 81, 2, 935-939.

WELCH, R. B. 1978. Perceptual modification: Adapting to altered sensory environments. Academic Press New York.

WELCH, R. B. 1986. Adaptation of space perception. Handbook of perception and human performance. 1, 21-24.

Welch, R. B., Bridgeman, B., AnAND, S., AND Browman, K. E. 1993. Alternating prism exposure causes dual adaptation and generalization to a novel displacement. Perception \& Psychophysics 54, 2, 195-204.

WelCh, R. B. AND SAMPANES, A. C. 2008. Adapting to virtual environments: visual-motor skill acquisition versus perceptual recalibration. Displays 29, 2, 152-158.

YUAN, Y. AND STEED, A. 2010. Is the rubber hand illusion induced by immersive virtual reality? 2010 IEEE Virtual Reality Conference (VR), 95-102.

Received February 2009; revised July 2009; accepted October 2009 


\title{
Online Appendix to: The effects of visuomotor calibration to the perceived space and body, through embodiment in immersive virtual reality.
}

\author{
ELENA KOKKINARA ${ }^{\mathrm{a}, \mathrm{b}, \mathrm{c}}$, MEL SLATER $^{\mathrm{a}, \mathrm{c}, \mathrm{d}}$ and JOAN LÓPEZ-MOLINER ${ }^{\mathrm{b}, \mathrm{c}}$ \\ ${ }^{a}$ EVENT Lab, Facultat de Psicologia, Universitat de Barcelona, Barcelona, Spain \\ ${ }^{b}$ VISCA lab, Departament de Psicologia Básica, Universitat de Barcelona, Spain \\ ${ }^{\mathrm{c}}$ Institute for Brain, Cognition and Behaviour (IR3C), Spain \\ dinstituci Catalana Recerca i Estudis Avancats (ICREA), Barcelona, Spain
}

\section{Technical characteristics}

The nVisor SX111 HMD has visual contrast $<100: 1$ and brightness $=5 \mathrm{fL}$, whereas the virtual room developed in Unity3D was illuminated with a directional light [intensity (multiplied by the light color) $=0.3$ ] and two point lights placed inside the virtual room [intensity (multiplied by the light color $)=0.43$ and 0.37$]$. The chosen light color of was $\operatorname{RGB}(255,255,236)$ for directional light and RGB $(255,255,236)$ the point lights. The update rate of the rendering of the virtual scene was $\sim 60 \mathrm{fps}$. Due to the complexity of the setup, the overall latency was not possible to measure. Tracking latency of the 6DOF tracker inside the 12-camera optical motion capture system to the Tracking Tools software was negligible $(\sim 4 \mathrm{~ms})$. The latency of the video streamed into the HDM was below $100 \mathrm{~ms}$. We measured the time lag of a light switching on in the HMD with respect to the switching on of an optical LED, activated from the Unity3D software via an Arduino Uno (assuming negligible latency), to be $\sim 80 \mathrm{~ms}$.

Further technical characteristics about nVisor SX111 can be found here:

http://www.bienetec.com/sites/default/files/nvisorsx111.pdf

(C) 2014 ACM 1544-3558/2014/05-ART1 $\$ 15.00$

DOI 10.1145/0000000.0000000 http://doi.acm.org/10.1145/0000000.0000000 
Table III. The Post Experience Questionnaire. All questions were on a Likert Scale from 1 (Totally disagree) to 7 (Totally agree).

\begin{tabular}{|l|l|l|}
\hline Question & Variable Name & Statement \\
\hline Q1 & q1location & During the game I felt as if my arm was located where I saw the virtual arm to be. \\
\hline Q2 & q2ownership & During the game I felt that the virtual arm was my own arm. \\
\hline Q3 & q3agency & The movements of the virtual arm were caused by my movements. \\
\hline Q4 & q4morearms & It seemed as if I might have more than two arms. \\
\hline Q5 & q5otherarms & During the game I felt that the virtual arm belonged to someone else. \\
\hline
\end{tabular}

Table IV. Mean and SD for Height drift measurments

\begin{tabular}{|l|l|l|l|l|}
\hline $\begin{array}{l}\text { Manipulatiaon } \\
\text { Type }\end{array}$ & $\begin{array}{l}\text { Manipulation } \\
\text { Extent }\end{array}$ & $\mathrm{N}$ & Mean & SD \\
\hline Spatial & Baseline & 16 & 0.031 & 0.047 \\
\hline Spatial & Congruent & 16 & 0.044 & 0.033 \\
\hline Spatial & Amplified & 16 & 0.059 & 0.037 \\
\hline Spatial & mAmplified & 16 & 0.059 & 0.033 \\
\hline Spatiotemporal & Baseline & 15 & 0.026 & 0.052 \\
\hline Spatiotemporal & Congruent & 15 & 0.018 & 0.021 \\
\hline Spatiotemporal & Amplified & 15 & 0.038 & 0.032 \\
\hline Spatiotemporal & mAmplified & 15 & 0.064 & 0.042 \\
\hline
\end{tabular}

Table V. Mean and SD for Width drift measurments

\begin{tabular}{|l|l|l|l|l|}
\hline $\begin{array}{l}\text { Manipulatiaon } \\
\text { Type }\end{array}$ & $\begin{array}{l}\text { Manipulation } \\
\text { Extent }\end{array}$ & $\mathrm{N}$ & Mean & SD \\
\hline Spatial & Baseline & 16 & 0.120 & 0.111 \\
\hline Spatial & Congruent & 16 & 0.180 & 0.084 \\
\hline Spatial & Amplified & 16 & 0.223 & 0.079 \\
\hline Spatial & mAmplified & 16 & 0.222 & 0.082 \\
\hline Spatiotemporal & Baseline & 15 & 0.089 & 0.066 \\
\hline Spatiotemporal & Congruent & 15 & 0.096 & 0.046 \\
\hline Spatiotemporal & Amplified & 15 & 0.126 & 0.036 \\
\hline Spatiotemporal & mAmplified & 15 & 0.169 & 0.083 \\
\hline
\end{tabular}

Table VI. Median and IQR for questionnaire

\begin{tabular}{|l|l|l|l|l|l|l|l|l|l|l|l|l|l|}
\hline $\begin{array}{l}\text { Manipulatiaon } \\
\text { Type }\end{array}$ & $\begin{array}{l}\text { Manipulation } \\
\text { Extent }\end{array}$ & $\mathrm{N}$ & \multicolumn{2}{|c|}{ q1location } & \multicolumn{2}{|c|}{ q20wnership } & \multicolumn{2}{|c|}{ q3agency } & \multicolumn{2}{c|}{ q4morearms } & \multicolumn{2}{c|}{ q50therarms } \\
\hline & & & Median & IQR & Median & IQR & Median & IQR & Median & IQR & Median & IQR \\
\hline Spatial & Congruent & 16 & 6.5 & 1.0 & 6 & 1.0 & 7.0 & 1.0 & 1.0 & 0.0 & 2 & 1.0 \\
\hline Spatial & Amplified & 16 & 7.0 & 2.0 & 6 & 2.0 & 7.0 & 1.25 & 1.0 & 0.0 & 1 & 1.0 \\
\hline Spatial & mAmplified & 16 & 6.0 & 2.0 & 6 & 0.5 & 6.5 & 2.00 & 1.0 & 2.25 & 2 & 3.0 \\
\hline Spatiotemporal & Congruent & 15 & 6.0 & 1.5 & 5 & 2.0 & 7.0 & 0.5 & 1.0 & 1.0 & 2.0 & 1.0 \\
\hline Spatiotemporal & Amplified & 15 & 4.0 & 1.0 & 5 & 2.5 & 6.0 & 2.5 & 1.0 & 1.5 & 4 & 3.5 \\
\hline Spatiotemporal & mAmplified & 15 & 4.0 & 3.0 & 5 & 2.5 & 5.0 & 2.5 & 1.0 & 1.5 & 3 & 4.0 \\
\hline
\end{tabular}




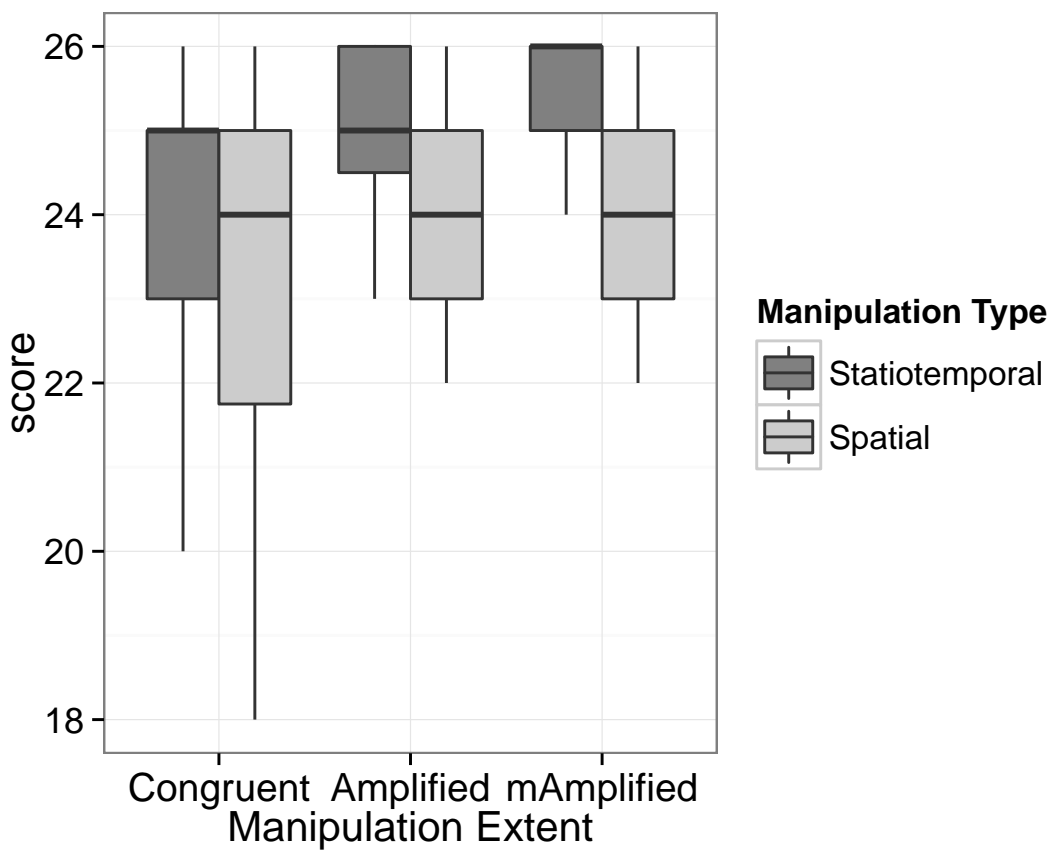

Fig. 8. Number of successful hits (score) during the targeting game. The overall number of targets on each trial is 26. In both spatiotemporal and spatial manipulations the score is significantly increasing over the three trials [Spatiotemporal: $(\mathrm{F}[2,28]=5.32, \mathrm{p}=0.01)$, post-hoc: Congruent-Amplified: $\mathrm{p}=0.08$, Congruent-mAmplified: $\mathrm{p}=0.0037$, AmplifiedmAmplified: $p=0.53$ ] [Spatial: $F[2,30]=2.4, p=0.108$ ), post-hoc: Congruent-Amplified: $p=0.1$ and Congruent-mAmplified: $p=0.2$, Amplified-mAmplified: $\mathrm{p}=0.95$ ] (two-tailed, using FDR corrections). Overall higher score was found in the spatiotemporal manipulation $(\mathrm{F}[1,29]=9.19, \mathrm{p}=0.005)$. 